\title{
M-MRAC With Normalization
}

\author{
Vahram Stepanyan and Kalmanje Krishnakumar
}

\begin{abstract}
This paper presents a normalization based modified reference model adaptive control method for multi-input multi-output (MIMO) uncertain systems in the presence of bounded external disturbances. It has been shown that desired tracking performance can be achieved for the system's output and input signals with the proper choice of design parameters. The resulting adaptive control signal satisfies a second order linear time invariant (LTI) system, which is the effect of the normalization term in the adaptive laws. This LTI system provides the guideline for the design parameter selection. The theoretical findings are confirmed via a simulation example.
\end{abstract}

\section{INTRODUCTION}

Adaptive control, and model reference adaptive control (MRAC) in particular, can provide a desired asymptotic performance for many uncertain systems. However, the transient performance of these systems is hard to quantify and regulate due to their inherent nonlinear nature. There have been many efforts to improve the transient of the tracking error (see for example recent results in [6], [14], [16], [25]), but not the control signal, the behavior of which significantly contributes to the limitations of the conventional adaptive methods (see for example [2]), thus preventing them from being widely adopted in safety-critical systems.

Recently, $\mathcal{L}_{1}$ adaptive control framework [8] had been introduced, which addresses the limitations of the adaptive control design by introducing a low-pass filter to the control channel. This sets a bandwidth a priori within which the uncertainties in the system can be compensated for.

An alternative method, modified reference model MRAC or M-MRAC for short, had been introduced in [23]. It is based on the modification of the reference model by the tracking error feedback, thus preventing the system's attempt to aggressively maneuver toward the reference model in the initial stage of the process. This approach has a systematic design guideline and is easy to implement, yet it can guarantee desired asymptotic and transient properties for the system's input and output signals. This method has been picked up by other researchers and used under different names. For example, in [7] it is called closed-loop reference model MRAC, while in [13] it is called adaptive control with observer-like reference model.

On the other hand, normalization has been used in adaptive control schemes to provide global boundedness and to improve robustness with respect to un-modeled dynamics and

Vahram Stepanyan is with University Affiliated Research Center, University of California Santa Cruz, Moffett Field, CA 94035, email: vahram.stepanyan@nasa.gov

Kalmanje Krishnakumar is with Intelligent Systems Division, NASA Ames Research Center, Moffett Field, CA 94035, email: kalmanje.krishnakumarenasa.gov bounded disturbances (see for example [5], [21], [9], [10], [11], [15], [18], [17]). Recently, normalized adaptive laws were applied to stabilization of uncertain systems using a logarithmic Lyapunov function [1], and to the design of a certainty equivalent controller [4].

In this paper we apply the normalized adaptive laws to $\mathrm{M}$ MRAC design for MIMO uncertain nonlinear systems in the presence of bounded disturbances. The parameter estimates are generated using a state prediction model, which incorporates a prediction error feedback term. The desired closedloop behavior is achieved with fast adaptation by selecting a proper error feedback gain, which also separates the time scale of the adaptive estimation from that of the system's dynamics. The normalization benefit is in the resulting LTI dynamics for the control signal, for which the error feedback gain determines the damping ratio and the adaptation rate determines the frequency. The rest of the paper outlines the properties of the proposed normalized M-MRAC architecture and demonstrates its benefits in a simulation example.

\section{Problem Statement}

Let the uncertain system be given by

$$
\dot{\boldsymbol{x}}(t)=A_{0} \boldsymbol{x}(t)+B_{0}[\boldsymbol{u}(t)+W \boldsymbol{f}(\boldsymbol{x}(t))+\boldsymbol{d}(t)]
$$

with $\boldsymbol{x}(0)=\boldsymbol{x}_{0}$, where $\boldsymbol{x} \in \mathbb{R}^{n}$ and $\boldsymbol{u} \in \mathbb{R}^{p}$ are the state and control of the system, $\boldsymbol{f}: \mathbb{R}^{n} \rightarrow \mathbb{R}^{q}$ is a vector of known functions assumed to be globally Lipschitz and $W \in \mathbb{R}^{p \times q}$ is an unknown constant matrix. The external disturbance $\boldsymbol{d}: \mathbb{R} \rightarrow \mathbb{R}^{p}$ is bounded and has a bounded derivative. $A_{0} \in \mathbb{R}^{n \times n}$ and $B_{0} \in \mathbb{R}^{n \times p}$ are unknown constant matrices satisfying the following matching conditions.

Assumption 2.1: Given a Hurwitz matrix $A \in \mathbb{R}^{n \times n}$ and a matrix $B \in \mathbb{R}^{n \times p}$ of full column rank, there exists a matrix $K_{1} \in \mathbb{R}^{p \times n}$ and a sign definite matrix $\Lambda \in \mathbb{R}^{p \times p}$ such that

$$
\begin{aligned}
& B_{0}=B \Lambda \\
& A_{0}=A+B K_{1} .
\end{aligned}
$$

Remark 2.1: The sign definiteness of $\Lambda$ corresponds to the conventional condition on the high frequency gain matrix of MIMO systems (see for example [18]). Without loss of generality we assume that $\Lambda$ is positive definite. The rest of the conditions for the existence of an adaptive controller are given by the equations (2). Systems in the form of (1) frequently arise in aerospace applications (see for example [12], [26] for diagonal $\Lambda$ ) and in robotics.

According to Assumption 2.1, the system (1) can be represented in the form

$$
\dot{\boldsymbol{x}}(t)=A \boldsymbol{x}(t)+B \Lambda\left[\boldsymbol{u}(t)+K_{1} \boldsymbol{x}(t)+W \boldsymbol{f}(\boldsymbol{x})+\boldsymbol{d}(t)\right] .
$$


It follows from the representation (3) that the nominal closed-loop system can be given by

$$
\dot{\boldsymbol{x}}_{m}(t)=A \boldsymbol{x}_{m}(t)+B \boldsymbol{r}(t)
$$

where $A, B$ satisfy the performance specifications, and $\boldsymbol{r}: R^{+} \rightarrow R^{q}$ is a bounded external command with a bounded derivative. This system with some initial condition $\boldsymbol{x}_{m}(0)=\boldsymbol{x}_{m 0}$ is selected as a reference model to be tracked. We introduce notations $K_{2}=-\Lambda^{-1}$ (which exists since $\Lambda$ is positive definite), $\boldsymbol{\phi}(t)=\left[\begin{array}{lll}\boldsymbol{f}^{\top}(\boldsymbol{x}(t)) & \boldsymbol{x}^{\top}(t) & \boldsymbol{r}^{\top}(t)\end{array}\right]^{\top}$, $\Theta=\left[\begin{array}{lll}W & K_{1} & K_{2}\end{array}\right]$ and write the system's dynamics as

$$
\dot{\boldsymbol{x}}(t)=A \boldsymbol{x}(t)+B \boldsymbol{r}(t)+B \Lambda[\boldsymbol{u}(t)+\Theta \boldsymbol{\phi}(t)+\boldsymbol{d}(t)] .
$$

Let $\boldsymbol{e}(t)=\boldsymbol{x}(t)-\boldsymbol{x}_{m}(t)$ be the tracking error. Then

$$
\dot{\boldsymbol{e}}(t)=A \boldsymbol{e}(t)+B \Lambda[\boldsymbol{u}(t)+\Theta \phi(t)+\boldsymbol{d}(t)]
$$

\section{CONTRol Design}

To estimate the unknown quantities we introduce the following adaptive prediction model

$$
\begin{aligned}
\dot{\hat{\boldsymbol{x}}}(t) & =A \boldsymbol{x}(t)+B \boldsymbol{r}(t) \\
& +B \hat{\Lambda}(t)[\boldsymbol{u}(t)+\hat{\Theta}(t) \boldsymbol{\phi}(t)+\hat{\boldsymbol{d}}(t)]+k \tilde{\boldsymbol{x}}(t)
\end{aligned}
$$

with $\hat{\boldsymbol{x}}(0)=\hat{\boldsymbol{x}}_{0}$, where $\tilde{\boldsymbol{x}}(t)=\boldsymbol{x}(t)-\hat{\boldsymbol{x}}(t)$ is the prediction error, $k>0$ is a design parameter and $\hat{\Lambda}(t), \hat{\Theta}(t)$ and $\hat{\boldsymbol{d}}(t)$ are the adaptive estimates of the unknown quantities. In this prediction model we use the term $A \boldsymbol{x}(t)$ instead of $A \hat{\boldsymbol{x}}(t)$ (as in [24]), which simplifies the stability analysis.

It follows from the system's dynamics (5) that designing the control signal as

$$
\boldsymbol{u}(t)=-\Theta \phi(t)-\boldsymbol{d}(t)
$$

translates the system into the reference model, achieving the control objective. Since $\boldsymbol{u}(t)$ is not implementable, its adaptive version

$$
\hat{\boldsymbol{u}}(t)=-\hat{\Theta}(t) \boldsymbol{\phi}(t)-\hat{\boldsymbol{d}}(t)
$$

is used to control the system. This control design reduces the prediction model to

$$
\dot{\hat{\boldsymbol{x}}}(t)=A \boldsymbol{x}(t)+B \boldsymbol{r}(t)+k \tilde{\boldsymbol{x}}(t)
$$

implying that there is no need to estimate $\Lambda$. The prediction error $\tilde{\boldsymbol{x}}(t)$ satisfies the dynamics

$$
\dot{\tilde{\boldsymbol{x}}}(t)=-k \tilde{\boldsymbol{x}}(t)+B \Lambda[\tilde{\Theta}(t) \boldsymbol{\phi}(t)+\overline{\boldsymbol{d}}(t)+\tilde{\boldsymbol{d}}(t)],
$$

where $\overline{\boldsymbol{d}}(t)=\boldsymbol{d}(t)-\boldsymbol{d}_{0}$ for some constant vector $\boldsymbol{d}_{0}$ (can be the average value of $\boldsymbol{d}(t))$, and $\tilde{\Theta}(t)=\Theta-\hat{\Theta}(t), \tilde{\boldsymbol{d}}(t)=$ $\boldsymbol{d}_{0}-\hat{\boldsymbol{d}}(t)$ are the estimation errors. The adaptive laws are introduced using normalization and the projection operators assuming that the bounds $\|\Theta\| \leq \vartheta^{*},\|\overline{\boldsymbol{d}}(t)\|_{\mathcal{L}_{\infty}} \leq d^{*}$ are known

$$
\begin{aligned}
& \dot{\hat{\Theta}}(t)=\operatorname{Pr}\left(\hat{\Theta}(t), \gamma \frac{B^{\top} \tilde{\boldsymbol{x}}(t) \boldsymbol{\phi}^{\top}(t)}{1+\boldsymbol{\phi}^{\top}(t) \boldsymbol{\phi}(t)}\right) \\
& \dot{\hat{\boldsymbol{d}}(t)}=\operatorname{Pr}\left(\hat{\boldsymbol{d}}(t), \gamma \frac{B^{\top} \tilde{\boldsymbol{x}}(t)}{1+\boldsymbol{\phi}^{\top}(t) \boldsymbol{\phi}(t)}\right),
\end{aligned}
$$

where $\gamma>0$ is the adaptation rate, $\operatorname{Pr}(\cdot, \cdot)$ denotes the projection operator [20].

\section{StabiLity}

Theorem 4.1: Let the system (5) be controlled by the adaptive control $\hat{\boldsymbol{u}}(t)$ defined by (9), (10) and (12). Then the closed loop signals are bounded. Moreover, if the disturbance $\boldsymbol{d}(t)$ is constant, then $\tilde{\boldsymbol{x}}(t) \rightarrow 0, \tilde{\boldsymbol{u}}(t) \rightarrow 0$ and $\boldsymbol{e}(t) \rightarrow 0$ as $t \rightarrow \infty$, where $\tilde{\boldsymbol{u}}(t)=\boldsymbol{u}(t)-\hat{\boldsymbol{u}}(t)$ is the control error.

Proof: The derivative of the candidate Lyapunov function

$$
\begin{aligned}
V(t) & =\frac{1}{2} \frac{\tilde{\boldsymbol{x}}^{\top}(t) \tilde{\boldsymbol{x}}(t)}{1+\boldsymbol{\phi}^{\top}(t) \boldsymbol{\phi}(t)}+(2 \gamma)^{-1} \tilde{\boldsymbol{d}}^{\top}(t) \Lambda \tilde{\boldsymbol{d}}(t) \\
& +(2 \gamma)^{-1} \operatorname{tr}\left(\tilde{\Theta}^{\top}(t) \Lambda \tilde{\Theta}(t)\right),
\end{aligned}
$$

computed along the trajectories of (11) and (12) has the form

$$
\begin{aligned}
\dot{V}(t) & =-k \frac{\tilde{\boldsymbol{x}}^{\top}(t) \tilde{\boldsymbol{x}}(t)}{1+\boldsymbol{\phi}^{\top}(t) \boldsymbol{\phi}(t)}+\frac{\tilde{\boldsymbol{x}}^{\top}(t) \tilde{\boldsymbol{x}}(t) \boldsymbol{\phi}^{\top}(t) \dot{\boldsymbol{\phi}}(t)}{\left(1+\boldsymbol{\phi}^{\top}(t) \boldsymbol{\phi}(t)\right)^{2}} \\
& +\frac{\tilde{\boldsymbol{x}}^{\top}(t) B \Lambda[\tilde{\Theta}(t) \boldsymbol{\phi}(t)+\overline{\boldsymbol{d}}(t)+\tilde{\boldsymbol{d}}(t)]}{1+\boldsymbol{\phi}^{\top}(t) \boldsymbol{\phi}(t)} \\
& +\gamma^{-1} \operatorname{tr}\left(\dot{\tilde{\Theta}}^{\top}(t) \Lambda \tilde{\Theta}(t)\right)+\gamma^{-1} \dot{\tilde{\boldsymbol{d}}}^{\top}(t) \Lambda \tilde{\boldsymbol{d}}(t)
\end{aligned}
$$

which upon substitution of the adaptive laws and application of the properties of projection operator reduces to the inequality

$$
\begin{aligned}
\dot{V}(t) & \leq-\frac{\tilde{\boldsymbol{x}}^{\top}(t) \tilde{\boldsymbol{x}}(t)}{1+\boldsymbol{\phi}^{\top}(t) \boldsymbol{\phi}(t)}\left(k-\frac{\boldsymbol{\phi}^{\top}(t) \dot{\boldsymbol{\phi}}(t)}{1+\boldsymbol{\phi}^{\top}(t) \boldsymbol{\phi}(t)}\right) \\
& +\frac{\tilde{\boldsymbol{x}}^{\top}(t) B \Lambda \overline{\boldsymbol{d}}(t)}{1+\boldsymbol{\phi}^{\top}(t) \boldsymbol{\phi}(t)} .
\end{aligned}
$$

To upper bound the sign indefinite term $\phi^{\top}(t) \dot{\phi}(t)$, we first notice that it follows from the Lipschitz condition that there exist positive constants $l_{1}, l_{2}$ such that $\|\boldsymbol{f}(\boldsymbol{x})\| \leq$ $l_{1}+l_{2}\|\boldsymbol{x}\|$ and $\|\dot{\boldsymbol{f}}(\boldsymbol{x})\| \leq l_{2}\|\dot{\boldsymbol{x}}\|$. Next, it follows from the system's dynamics that $\|\dot{\boldsymbol{x}}(t)\| \leq\|A\|\|\boldsymbol{x}(t)\|+\|B\|\|\boldsymbol{r}(t)\|+$ $\|B \Lambda\|\left[\|\tilde{\Theta}(t)\|\left(l_{1}+l_{2}\|\boldsymbol{x}\|+\|\boldsymbol{x}\|+\|\boldsymbol{r}\|\right)+\|\tilde{\boldsymbol{d}}(t)\|\right]$. Since the projection operator in the adaptive laws (12) guarantees the inequalities $\|\hat{\Theta}(t)\| \leq \vartheta^{*}$ and $\|\hat{\boldsymbol{d}}(t)\| \leq d^{*}$, we have $\|\tilde{\Theta}(t)\| \leq 2 \vartheta^{*}$ and $\|\tilde{\boldsymbol{d}}(t)\| \leq 2 d^{*}$. Therefore, we obtain

$$
\|\dot{\boldsymbol{x}}(t)\| \leq c_{1}\|\boldsymbol{x}(t)\|+c_{2}\|\boldsymbol{r}(t)\|+c_{3},
$$

where the constants $c_{1}, c_{2}, c_{3}$ are readily computed. Now, we can write $\left\|\boldsymbol{\phi}^{\top}(t) \dot{\boldsymbol{\phi}}(t)\right\| \leq\left(l_{1}+l_{2}\|\boldsymbol{x}(t)\|\right) l_{2}\|\dot{\boldsymbol{x}}(t)\|+$ $\|\boldsymbol{x}(t)\|\|\dot{\boldsymbol{x}}(t)\|+\|\boldsymbol{r}(t)\|\|\dot{\boldsymbol{r}}(t)\|$. Substituting $\|\dot{\boldsymbol{x}}(t)\|$ and completing the squares, we obtain the following upper bound

$$
\left\|\boldsymbol{\phi}^{\top}(t) \dot{\phi}(t)\right\| \leq c_{4}\|\boldsymbol{x}(t)\|^{2}+c_{5}\|\boldsymbol{r}(t)\|^{2}+c_{6},
$$

where the positive constant $c_{4}, c_{5}, c_{6}$ are straightforward to compute. Denoting $\rho=\max \left(c_{4}, c_{5}, c_{6}\right)$, we arrive at

$\left\|\boldsymbol{\phi}^{\top}(t) \dot{\boldsymbol{\phi}}(t)\right\| \leq \rho\left(1+\|\boldsymbol{x}(t)\|^{2}+\|\boldsymbol{r}(t)\|^{2}\right) \leq \rho\left(1+\boldsymbol{\phi}^{\top}(t) \boldsymbol{\phi}(t)\right)$, which implies that

$\dot{V}(t) \leq-\frac{\|\tilde{\boldsymbol{x}}(t)\|}{1+\boldsymbol{\phi}^{\top}(t) \boldsymbol{\phi}(t)}\left[(k-\rho)\|\tilde{\boldsymbol{x}}(t)\|-d^{*}\|B \Lambda\|\right]$.

Selecting $k>\rho$ we conclude that $\dot{V}(t) \leq 0$ when $\|\tilde{\boldsymbol{x}}(t)\| \geq$ $d^{*}\|B \Lambda\|(k-\rho)^{-1}$. Therefore, $\tilde{\boldsymbol{x}}(t)$ is bounded. In order to 
show the boundedness of $\hat{\boldsymbol{x}}(t)$ we introduce an auxiliary model

$$
\dot{\boldsymbol{z}}(t)=A \boldsymbol{z}(t)+B \boldsymbol{r}(t)+k \tilde{\boldsymbol{x}}(t) .
$$

Since $\boldsymbol{r}(t)$ and $\tilde{\boldsymbol{x}}(t)$ are bounded and $A$ is Hurwitz, it follows that $\boldsymbol{z}(t)$ is bounded. Let $\tilde{\boldsymbol{z}}(t)=\hat{\boldsymbol{x}}(t)-\boldsymbol{z}(t)$. Clearly, the error signal $\tilde{\boldsymbol{z}}(t)$ satisfies the equation

$$
\dot{\tilde{\boldsymbol{z}}}(t)=A \tilde{\boldsymbol{z}}(t)+A \tilde{\boldsymbol{x}}(t) .
$$

Since $\tilde{\boldsymbol{x}}(t)$ is bounded, it follows that $\tilde{\boldsymbol{z}}(t), \hat{\boldsymbol{x}}(t), \boldsymbol{x}(t), \boldsymbol{\phi}(t)$ and $\hat{\boldsymbol{u}}(t)$ are bounded. When $\boldsymbol{d}(t)$ is constant $\left(\boldsymbol{d}(t)=\boldsymbol{d}_{0}\right)$, the inequality (15) reduces to

$$
\dot{V}(t) \leq-(k-\rho) \frac{\|\tilde{\boldsymbol{x}}(t)\|^{2}}{1+\boldsymbol{\phi}^{\top}(t) \boldsymbol{\phi}(t)},
$$

meaning that $\tilde{\boldsymbol{x}}(t) \in \mathcal{L}_{\infty}$ when $k>\rho$. Following the same steps as above we conclude that $\hat{\boldsymbol{x}}(t), \boldsymbol{x}(t), \boldsymbol{\phi}(t), \hat{\boldsymbol{u}}(t), \tilde{\boldsymbol{u}}(t), \dot{\tilde{\boldsymbol{x}}}(t) \in \mathcal{L}_{\infty}$. On the other hand, integrating the inequality (19) we obtain

$$
(k-\rho) \int_{0}^{t} \frac{\|\tilde{\boldsymbol{x}}(t)\|^{2}}{1+\boldsymbol{\phi}^{\top}(t) \phi(t)} \leq V(0),
$$

which implies that $\tilde{\boldsymbol{x}}(t) \in \mathcal{L}_{2}$, since $\phi(t) \in \mathcal{L}_{\infty}$. Application of Barbalat's lemma ([22], p.19) results in $\tilde{\boldsymbol{x}}(t) \rightarrow 0$ as $t \rightarrow$ $\infty$. Next, differentiating the prediction error dynamics (11) we observe that $\ddot{\widetilde{\boldsymbol{x}}}(t) \in \mathcal{L}_{\infty}$, implying that $\dot{\tilde{\boldsymbol{x}}}(t)$ is uniformly continuous. Since it has a bounded integral $\tilde{\boldsymbol{x}}(t)$, it follows that $\dot{\tilde{\boldsymbol{x}}}(t) \rightarrow 0$ as $t \rightarrow \infty$. Then, representing (11) as

$$
\dot{\tilde{\boldsymbol{x}}}(t)=-k \tilde{\boldsymbol{x}}(t)+B \Lambda \tilde{\boldsymbol{u}}(t),
$$

we conclude that $\tilde{\boldsymbol{u}}(t) \rightarrow 0$ as $t \rightarrow \infty$, since $B$ is of full column rank. Finally, writing the error dynamics (6) as

$$
\dot{\boldsymbol{e}}(t)=A \boldsymbol{e}(t)+B \Lambda \tilde{\boldsymbol{u}}(t)
$$

we see that $e(t) \rightarrow 0$ as $t \rightarrow \infty$. The proof is complete.

\section{TRANSIENT BOUNDS}

To obtain transient bounds we conduct a dynamic analysis of the control error $\tilde{\boldsymbol{u}}(t)$. To this end we notice that selection of the initial parameter estimates inside the convex sets defined by the projection operator results in

$$
\begin{aligned}
\dot{\hat{\Theta}}(t) & =\gamma \boldsymbol{\eta}(t) \phi^{\top}(t) \\
\dot{\hat{\boldsymbol{d}}}(t) & =\gamma \boldsymbol{\eta}(t)
\end{aligned}
$$

on some initial interval $\left[0 t_{1}\right]$. Therefore, $\dot{\tilde{\boldsymbol{u}}}(t)$ can be computed on that interval as follows

$$
\begin{aligned}
\dot{\tilde{\boldsymbol{u}}}(t) & =-\dot{\hat{\Theta}}(t) \boldsymbol{\phi}(t)+\tilde{\Theta}(t) \dot{\boldsymbol{\phi}}(t)+\dot{\boldsymbol{d}}(t)-\dot{\hat{\boldsymbol{d}}}(t) \\
& =-\gamma B^{\top} \tilde{\boldsymbol{x}}(t)+\boldsymbol{h}(t),
\end{aligned}
$$

where $\boldsymbol{h}(t)=\tilde{\Theta}(t) \dot{\boldsymbol{\phi}}(t)+\dot{\boldsymbol{d}}(t)$ is bounded according to Theorem 4.1. That is there exists $\alpha>0$ such that $\|\boldsymbol{h}(t)\|_{\mathcal{L}_{\infty}} \leq \alpha$. Using the prediction error dynamics (21) it is straightforward to verify that

$$
\ddot{\tilde{\boldsymbol{u}}}(t)+k \dot{\tilde{\boldsymbol{u}}}(t)+\gamma B^{\top} B \Lambda \tilde{\boldsymbol{u}}(t)=k \boldsymbol{h}(t)+\dot{\boldsymbol{h}}(t) .
$$

Since $B^{\top} B$ and $\Lambda$ are symmetric and positive definite, there exists a nonsingular matrix $T$ such that $\Sigma=T B^{\top} B T^{\top}$ is diagonal with positive entries $\sigma_{i}, i=1, \ldots, p$ and $T^{-\top} \Lambda T^{-1}=\mathbb{I}$ [3] (p.245). Then, $\Sigma=T B^{\top} B T^{\top}=$ $T B^{\top} B T^{\top} T^{-\top} \Lambda T^{-1}=T B^{\top} B \Lambda T^{-1}$. Therefore, (25) can be written component-wise in a new variable $\boldsymbol{v}(t)=T \tilde{\boldsymbol{u}}(t)$ as

$$
\ddot{v}_{i}(t)+k \dot{v}_{i}(t)+\gamma \sigma_{i} v_{i}(t)=k \bar{h}_{i}(t)+\dot{\bar{h}}_{i}(t),
$$

where $\overline{\boldsymbol{h}}(t)=T \boldsymbol{h}(t)$. The equation (26) is linear time invariant, which is the benefit of using normalized adaptive laws. Applying the Laplace transform we obtain

$v_{i}(s)=g_{1 i}(s)\left[\bar{h}_{i}(s)+v_{i}(0)\right]+g_{2 i}(s)\left[\dot{v}_{i}(0)-\bar{h}_{i}(0)\right]$

where $g_{1 i}(s)=\frac{s+k}{s^{2}+k s+\gamma \sigma_{i}}$ and $g_{2 i}(s)=\frac{1}{s^{2}+k s+\gamma \sigma_{i}}$. It is easy to see that $\dot{\boldsymbol{v}}(0)-\overline{\boldsymbol{h}}(0)=T[\tilde{\boldsymbol{v}}(0)-\boldsymbol{h}(0)]=$ $-\gamma T B^{\top} \tilde{\boldsymbol{x}}(t) \equiv-\gamma \boldsymbol{a}$. Therefore,

$$
v_{i}(s)=g_{1 i}(s)\left[\bar{h}_{i}(s)+v_{i}(0)\right]-\gamma g_{2 i}(s) a_{i} .
$$

Theorem 5.1: Let the system (5) be controlled by the controller (9), which is defined by the prediction model (7) and the adaptive law (12). Then

$$
\begin{aligned}
\|\tilde{\boldsymbol{u}}(t)\| & \leq \beta_{1} e^{-\frac{k}{2} t}+\frac{\beta_{2}}{\sqrt{\gamma}} \\
\|\tilde{\boldsymbol{x}}(t)\| & \leq \beta_{3} e^{-k t}+\frac{\beta_{4}}{\sqrt{\gamma}} \\
\|\boldsymbol{e}(t)\| & \leq \beta_{5} e^{-\nu t}+\frac{\beta_{6}}{\sqrt{\gamma}},
\end{aligned}
$$

where $\nu$ and $\beta_{i}, i=1, \ldots, 6$ are positive constants to be specified in the proof.

Proof: According to [23], if we select $k=\sqrt{2 \gamma \sigma_{i}}$, then for all $i=1, \ldots, p$

$$
\left\|g_{1 i}(s)\right\|_{\mathcal{H}_{\infty}}=\sqrt{\frac{\alpha_{0}}{\gamma \sigma_{i}}}, \quad\left\|g_{2 i}(s)\right\|_{\mathcal{H}_{\infty}}=\frac{1}{\gamma \sigma_{i}} .
$$

where $\alpha_{0}=\sqrt{\frac{\sqrt{5}+2}{2}}$. Let $\sigma_{0}=\min \left(\sigma_{i}, i=1, \ldots, p\right)$ and $\sigma^{0}=\max \left(\sigma_{i}, i=1, \ldots, p\right)$. It can be shown that if we set

$$
k=\sqrt{2 \gamma \sigma^{0}}
$$

then $\left\|g_{1 i}(s)\right\|_{\mathcal{H}_{\infty}} \leq \sqrt{\frac{\alpha_{0}}{\gamma \sigma_{0}}}, \quad\left\|g_{2 i}(s)\right\|_{\mathcal{H}_{\infty}} \leq \frac{1}{\gamma \sigma_{0}}$ for all $i=$ $1, \ldots, p$. It follows from (27) that $v_{i}(t)$ can be bounded as

$$
\left\|v_{i}(t)\right\|_{\mathcal{L}_{\infty}} \leq \frac{\sqrt{\alpha_{0}}\left\|\bar{h}_{i}(t)-v_{i}(0)\right\|_{\mathcal{L}_{\infty}}}{\sqrt{\gamma \sigma_{0}}}+\frac{\left|a_{i}\right|}{\sigma_{0}},
$$

which implies that

$$
\|\boldsymbol{v}(t)\|_{\mathcal{L}_{\infty}} \leq \frac{\alpha \sqrt{\alpha_{0}}+\|\boldsymbol{v}(0)\| \sqrt{\alpha_{0}}}{\sqrt{\gamma \sigma_{0}}}+\frac{\|a\|}{\sigma_{0}} .
$$

Since $T$ is nonsingular, we have $\|\tilde{\boldsymbol{u}}(t)\|=\left\|T^{-1} \boldsymbol{v}(t)\right\| \leq$ $\left\|T^{-1}\right\|\|\boldsymbol{v}(t)\|=\|T\|^{-1}\|\boldsymbol{v}(t)\|$. Therefore,

$$
\|\tilde{\boldsymbol{u}}(t)\|_{\mathcal{L}_{\infty}} \leq \frac{\alpha \sqrt{\alpha_{0}}+\|T \tilde{\boldsymbol{u}}(0)\| \sqrt{\alpha_{0}}}{\|T\| \sqrt{\gamma \sigma_{0}}}+\frac{\left\|B^{\top} \tilde{\boldsymbol{x}}(0)\right\|}{\sigma_{0}}
$$


Since LTI system (25) is exponentially stable, the effect of the initial conditions $\tilde{\boldsymbol{u}}(0)$ and $\dot{\tilde{\boldsymbol{u}}}(0)$ exponentially die out with the rate $k / 2$. Therefore, the first inequality (29) holds with $\beta_{1}=\frac{\|\tilde{\boldsymbol{u}}(0)\| \sqrt{\alpha_{0}}}{\sqrt{\gamma \sigma_{0}}}+\frac{\left\|B^{\top} \tilde{\boldsymbol{x}}(0)\right\|}{\sigma_{0}}$ and $\beta_{2}=\frac{\alpha \sqrt{\alpha_{0}}}{\|T\| \sqrt{\sigma_{0}}}$.

The bound of the prediction error $\tilde{\boldsymbol{x}}(t)$ is obtained by integrating the dynamics (21)

$$
\begin{aligned}
\|\tilde{\boldsymbol{x}}(t)\| & \leq\|\tilde{\boldsymbol{x}}(0)\| e^{-k t}+\frac{\|B \Lambda\|}{k}\left(1-e^{-k t}\right)\|\tilde{\boldsymbol{u}}(t)\|_{\mathcal{L}_{\infty}} \\
& \leq\|\tilde{\boldsymbol{x}}(0)\| e^{-k t}+\frac{\|B \Lambda\|}{k}\|\tilde{\boldsymbol{u}}(t)\|_{\mathcal{L}_{\infty}}
\end{aligned}
$$

which is in the form of the second inequality (29) with $\beta_{3}=$ $\|\tilde{\boldsymbol{x}}(0)\|$, and $\beta_{4}$ is readily computed using (33).

Finally, taking into account the fact that for the Hurwitz matrix $A$ there exist positive constants $c$ and $\nu$ such that $\left\|e^{A t}\right\| \leq c e^{-\nu t}$, the tracking error's bound is derived from the dynamics (22) by direct integration

$$
\|\boldsymbol{e}(t)\| \leq c\|\boldsymbol{e}(0)\| e^{-\nu t}+c\|B \Lambda\|\left\|A^{-1}\right\|\|\tilde{\boldsymbol{u}}(t)\|_{\mathcal{L}_{\infty}},
$$

which is the third inequality (29) with the constants similarly defined. The proof is complete.

Remark 5.1: It follows from Theorem 5.1 that the bound of the prediction error can be decreased by increasing either of the parameters $k$ and $\gamma$, whereas the bound of the tracking error can be decreased by increasing the adaptation rate $\gamma$. Also, the time scale of the tracking error dynamics is set by the reference model and cannot be altered by the choice of $k$, which on the other hand sets the time scale of the prediction dynamics. The control signal dynamics are affected by both design parameters. In particular, the damping ratio of the control signal is determined by $k$. Hence, the oscillations can be eliminated by the proper choice of $k$, which is given by (31). This choice of $k$ enables the designer to set the proper adaptation rate $\gamma$ taking into account the hardware bandwidth and the computational power limitations.

\section{Normalization EFFeCt}

The normalized adaptive law (12) can be viewed as an unnormalized adaptive law

$$
\begin{aligned}
\dot{\hat{\Theta}}(t) & =\operatorname{Pr}\left(\hat{\Theta}(t), \gamma_{1} B^{\top} \tilde{\boldsymbol{x}}(t) \boldsymbol{\phi}^{\top}(t)\right) \\
\dot{\hat{\boldsymbol{d}}}(t) & =\operatorname{Pr}\left(\hat{\boldsymbol{d}}(t), \gamma_{1} B^{\top} \tilde{\boldsymbol{x}}(t)\right),
\end{aligned}
$$

where the adaptation rate $\gamma_{1}=\frac{\gamma}{1+\phi^{\top}(t) \phi(t)}$ is variable. This does not change the stability proof of the prediction error. On the other hand, Theorem 5.1 defines the relationship between the feedback gain and adaptation rate as $k=$ $\sqrt{2 \gamma \lambda_{\max }\left(B^{\top} B\right)}=\sqrt{2 \gamma_{1} \lambda_{\max }\left(B^{\top} B\right)\left[1+\phi^{\top}(t) \phi(t)\right]}$. Therefore, instead of selecting the variable adaptation rate $\gamma_{1}$, which results into constant feedback gain $k$, one may select a constant $\gamma_{1}$, which results into a variable feedback gain $k$. That is, the effect of the normalized adaptive laws can be expressed in terms of a properly chosen variable gain for the error feedback term in the prediction model design, along with the unnormalized adaptive laws for the parameter estimation. This does not change the form of the prediction error dynamics. In this case the stability proof is simpler and can be conducted using a conventional Lyapunov function. However, the analysis of the control signal dynamics gets complicated. It is easy to see that $\dot{\tilde{\boldsymbol{u}}}(t)$ now takes the form

$$
\dot{\tilde{\boldsymbol{u}}}(t)=-\gamma_{1} \mu(t) B^{\top} \tilde{\boldsymbol{x}}(t)+\boldsymbol{h}(t),
$$

which differs from (24) by the factor $\mu(t)=1+\phi^{\top}(t) \phi(t)$ in the first term on the right hand side. The second derivative satisfies the equation

$$
\begin{aligned}
& \ddot{\tilde{\boldsymbol{u}}}(t)+k(t) \dot{\tilde{\boldsymbol{u}}}(t)+\gamma_{1} \mu(t) B^{\top} B \Lambda \tilde{\boldsymbol{u}}(t)= \\
& -\gamma_{1} \dot{\mu}(t) \mu(t) B^{\top} \tilde{\boldsymbol{x}}(t)+k(t) \boldsymbol{h}(t)+\dot{\boldsymbol{h}}(t),
\end{aligned}
$$

which again can be written in the decoupled form

$$
\begin{gathered}
\ddot{v}_{i}(t)+k(t) \dot{v}_{i}(t)+\gamma \sigma_{i} \mu(t) v_{i}(t)= \\
k(t) \bar{h}_{i}(t)+\overline{\bar{h}}_{i}(t)-\gamma_{1} \dot{\mu}(t) \mu(t) a_{i}(t),
\end{gathered}
$$

using transformation $\boldsymbol{v}(t)=T \tilde{\boldsymbol{u}}(t)$. Although all terms in (39) are bounded, $k(t)$ and $\gamma \sigma_{i} \mu(t)$ are positive definite, and the damping ration is $\zeta=\sqrt{\frac{\sigma^{0}}{2 \sigma_{i}}}$ as in the case of the equation (26), it is hard to make a conclusion about the bound on $v_{i}(t)$ due to time variant nature of the coefficients in (39). We will not pursue the derivation of this bound, but will numerically show in the simulation example, that M-MRAC design from [24] with variable error feedback gain is equivalent to $M-$ MRAC design with normalization.

\section{ILLUSTRATIVE EXAMPLE}

As a simulation example we consider a dynamic model that represents the lateral-directional motion of a generic transport aircraft (GTM) [19]. The nominal model is the linearized lateral-directional dynamics of GTM at the altitude of $30000 \mathrm{ft}$ and speed of $0.8 \mathrm{M}$ and is given by

$$
\dot{\boldsymbol{x}}(t)=A_{n} \boldsymbol{x}(t)+B_{n} \boldsymbol{u}(t),
$$

where $\boldsymbol{x}=\left[\begin{array}{llll}\beta & r & p & \phi\end{array}\right]^{\top}$ is the lateral-directional state vector, in which $\beta$ is the sideslip angle, $r$ is the yaw rate, $p$ is the roll rate, $\phi$ is the bank angle, and $\boldsymbol{u}=\left[\begin{array}{ll}\delta_{a} & \delta_{r}\end{array}\right]^{\top}$ is the control signal that includes the aileron deflection $\delta_{a}$ and the rudder deflection $\delta_{r}$, and the numerical values for $A_{n}$ and $B_{n}$ are

$$
\begin{gathered}
A_{n}=\left[\begin{array}{cccc}
-0.1578 & -0.9907 & 0.0475 & 0.0404 \\
2.7698 & -0.3842 & 0.0240 & 0 \\
-10.1076 & 0.5090 & -1.7520 & 0 \\
0 & 0.0506 & 1.0000 & 0
\end{array}\right], \\
B_{n}=\left[\begin{array}{cc}
0.0042 & 0.0476 \\
0.0351 & -2.2464 \\
6.3300 & 1.7350 \\
0 & 0
\end{array}\right] .
\end{gathered}
$$

The reference model is selected according to equation (4), with $A=A_{n}-B_{n} K, B=B_{n} N$, and

$$
K=\left[\begin{array}{cccc}
0 & 0 & 0.43 & 0.55 \\
1.92 & -1.5 & 0 & 0
\end{array}\right], N=\left[\begin{array}{cc}
1.26 & 0.65 \\
3.33 & -0.07
\end{array}\right] \text {. }
$$

The external command is a series of coordinated turn maneuvers. That is, sideslip angle command is set to zero and 

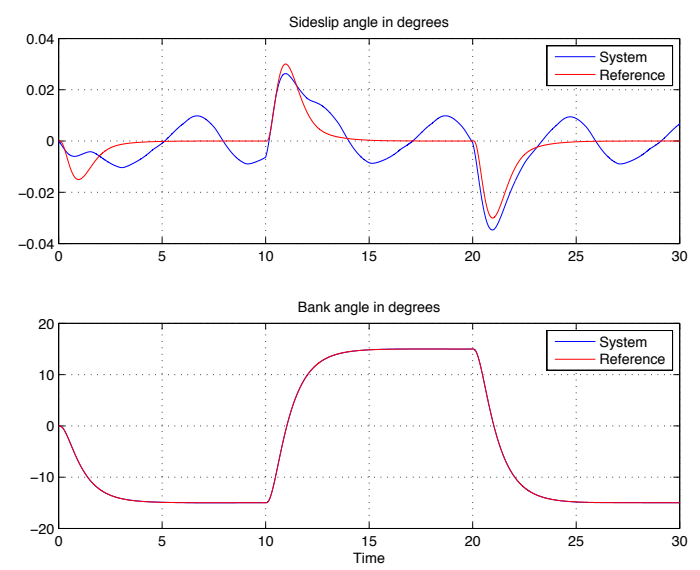

Fig. 1. Normalized M-MRAC output tracking performance.
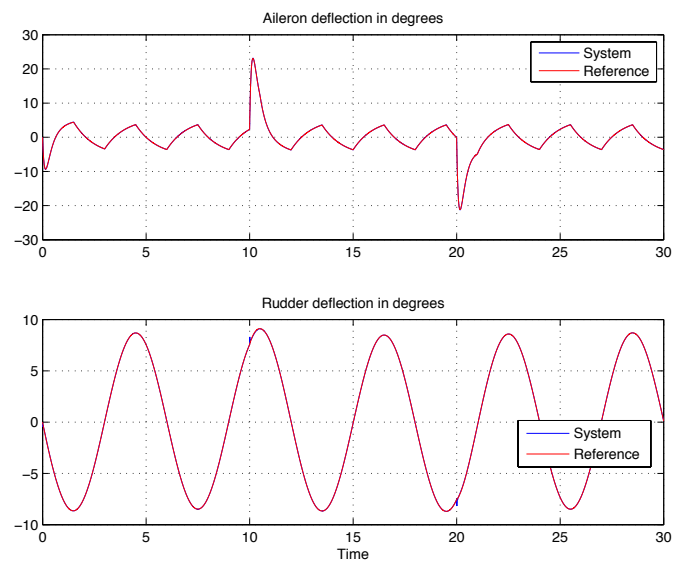

Fig. 2. Normalized M-MRAC input tracking performance.

the bank angle command is chosen to be a square wave of the amplitude of 15 degrees and of the frequency $\frac{\pi}{10} \mathrm{rad} / \mathrm{sec}$, which is filtered through a first order stable filter $\frac{10}{s+10}$.
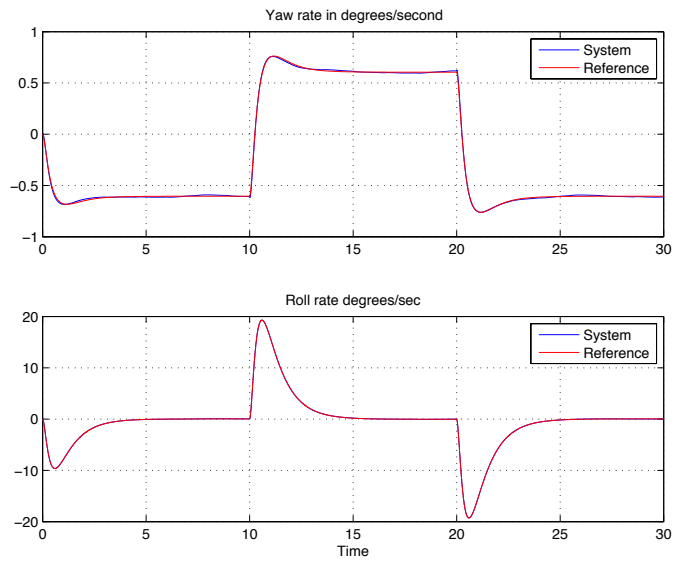

Fig. 3. Angular rate responses.

The uncertain model of GTM roughly corresponds to $28 \%$
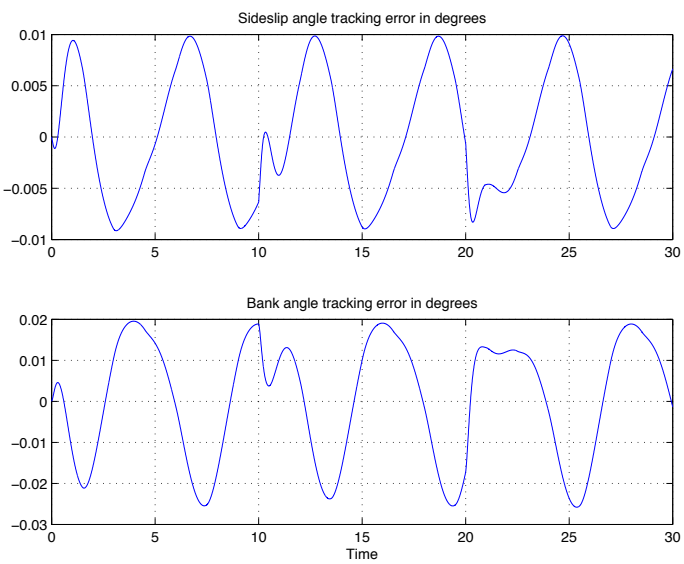

Fig. 4. Normalized M-MRAC tracking errors.

loss of left wing tip at $t=0 \mathrm{sec}$. Its dynamics are in the form of the equation (3) with $\boldsymbol{f}(\boldsymbol{x})=0$ and

$$
\begin{gathered}
\Theta=\left[\begin{array}{cccc}
-0.1820 & 0.0149 & -0.1049 & 0 \\
0.0807 & -0.0109 & 0.0168 & 0
\end{array}\right] \\
\Lambda=\left[\begin{array}{cc}
0.5401 & 0.0167 \\
-0.0632 & 1.0524
\end{array}\right] .
\end{gathered}
$$

The external disturbance is chosen to be a sinusoid of amplitude 0.1 and frequency $2 \pi / 3 \mathrm{rad} / \mathrm{sec}$ in the yaw channel and a filtered square wave of amplitude 0.15 and frequency $\pi / 3 \mathrm{rad} / \mathrm{sec}$ in the roll channel. The disturbance magnitudes correspond to 8.6 degrees of aileron deflection and 5.7 degrees of rudder deflection. In the definition of the projection operator the conservative bounds $\vartheta^{*}=d^{*}=10$ are used.

First, a simulation is run with $\gamma=5000$ and $k$ computed according to (31). Figure 1 displays the tracking performance of the states. Clearly good tracking is achieved (the sideslip angle is in hundredths of a degree) with the chosen gains, for which the control time history is presented in Figure 2. It can be observed that the adaptive control signal closely follows the reference signal given by (8). The angular rate performances are presented in Figure 3.

For the close examination we also display the tracking and control signal errors in Figure 4 and Figure 5. It can be observed that the tracking errors are negligible despite the significant disturbance, and the control signal errors exhibit spikes at instances when the command changes the direction.

For the comparison purposes we also run simulations with unnormalized M-MRAC and with the variable error feedback gain. Table I presents magnitudes of all error signals. It can be observed that unnormalized M-MRAC has a better performance in tracking and variable feedback gain $\mathrm{M}$ MRAC has a better performance in prediction. While the first is attributed to higher effective adaptation rate for the same $\gamma$ in unnormalized adaptive laws compared to normalized laws, the later is attributed to higher error feedback gain, which implies a faster prediction model. We notice that in this simulation example the states and the external commands 

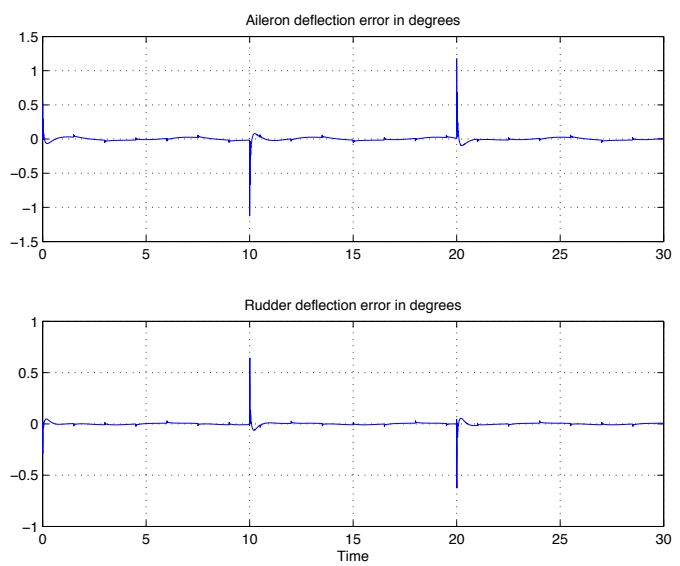

Fig. 5. Normalized M-MRAC control signal errors.

TABLE I

ERRORS MAGNITUDES FOR $\gamma=5000$.

\begin{tabular}{|c|c|c|c|}
\hline M-MRAC & Unnormalized & Normalized & Variable feedback gain \\
\hline$e_{\beta}(t)$ & 0.0099 & 0.0112 & 0.0105 \\
\hline$e_{\phi}(t)$ & 0.0258 & 0.0293 & 0.0274 \\
\hline$\tilde{\delta}_{a}(t)$ & 1.1796 & 1.2840 & 1.2124 \\
\hline$\tilde{\delta}_{r}(t)$ & 0.6418 & 0.6768 & 0.6381 \\
\hline$\tilde{\beta}(t)$ & 0.0002 & 0.0003 & 0.0002 \\
\hline$\tilde{\phi}(t)$ & 0.0001 & 0.0001 & 0.0001 \\
\hline$\tilde{r}(t)$ & 0.0093 & 0.0100 & 0.0091 \\
\hline$\tilde{p}(t)$ & 0.0163 & 0.0181 & 0.0162 \\
\hline
\end{tabular}

are small in magnitude (in simulation they were in radians), hence $\mu(t)$ is very close to unity, which results in comparable error signals in normalized and unnormalized M-MRAC.

Table II presents the error signal magnitudes, when the adaptation rate $\gamma$ is increased fourfold. It can be observed that all error signals are decreased about $50 \%$ as predicted by theoretical bounds.

\section{CONCLUSIONS}

We have presented a modified reference model MRAC approach with normalization for nonlinear uncertain systems in the presence of bounded external disturbances. The method results in predictable transient and asymptotic behavior for state and input variables of the system. The corresponding control signal obeys a second order LTI system, and can be regulated by the proper choice of design parameters.

TABLE II

ERRORS MAGNITUDES FOR $\gamma=20000$.

\begin{tabular}{|c|c|c|c|}
\hline M-MRAC & Unnormalized & Normalized & Variable feedback gain \\
\hline$e_{\beta}(t)$ & 0.0048 & 0.0055 & 0.0051 \\
\hline$e_{\phi}(t)$ & 0.0127 & 0.0145 & 0.0136 \\
\hline$\tilde{\delta}_{a}(t)$ & 0.6125 & 0.6550 & 0.6138 \\
\hline$\tilde{\delta}_{r}(t)$ & 0.3321 & 0.3514 & 0.3292 \\
\hline$\tilde{\beta}(t)$ & 0.0001 & 0.0001 & 0.0001 \\
\hline$\tilde{\phi}(t)$ & 0.0000 & 0.0000 & 0.0000 \\
\hline$\tilde{r}(t)$ & 0.0024 & 0.0026 & 0.0024 \\
\hline$\tilde{p}(t)$ & 0.0042 & 0.0046 & 0.0041 \\
\hline
\end{tabular}

\section{REFERENCES}

[1] S. Akhtar, R. Venugopal, and D. S. Bernstein, "Logarithmic Lyapunov Functions for Adaptive Stabilization with Normalized Adaptive Laws," International Journal of Control, vol. 77, no. 7, pp. 630-638, 2004.

[2] B. D. O. Anderson, "Failures of Adaptive Control Theory and Their Resolution," Communications in Information and Systems, vol. 5, no. 1, pp. 1-20, 2005.

[3] D. S. Bernstein, Matrix Mathematics. Princeton University Press, Princeton, NJ, 2005.

[4] J. D. Boskovic and Z. Han, "Certainty Equivalence Adaptive Control of Plants With Unmatched Uncertainty Using State Feedback," IEEE Trans. Autom. Contr, vol. 54, no. 8, pp. 1918 - 1924, 2009.

[5] B. Egardt, Stability of Adaptive Controllers. Springer-Verlag, Berlin, 1979 .

[6] B. Fidan, Y. Zhang, and P. Ioannou, "Adaptive Control of a Class of Slowly Time Varying Systems With Modeling Uncertainties," IEEE Trans. Autom. Contr., vol. 50, no. 6, pp. 915 - 920, 2005.

[7] T. E. Gibson, A. M. Annaswamy, and E. Lavretsky, "Adaptive systems with closed-loop reference-models, part I: Transient performance," In Proc. of the American Control Conference, Washington, DC, pp. 3376 - 3383, June 2013.

[8] N. Hovakimyan and C. Cao, L1 Adaptive Control Theory. Society for Industrial and Applied Mathematics, Philadelphia, PA, 2010.

[9] P. A. Ioannou and P. V. Kokotovic, "Instability Analysis and the Improvement of Robustness of Adaptive Controller," Automatica, vol. 20, no. 5, September 1989.

[10] P. A. Ioannou and S. Tsakalis, "A Robust Direct Adaptive Controller," IEEE Trans. Autom. Contr, vol. AC-31, pp. 1033-1043, 1986.

[11] G. Kreisselmeier and B. D. O. Anderson, "Robust Model Reference Adaptive Control," IEEE Trans. Autom. Contr., vol. AC-31, pp. 127$133,1986$.

[12] E. Lavretsky, "Combined/Composite Model Reference Adaptive Control," IEEE Trans. Autom. Contr., vol. 54, no. 11, pp. 2692-2697, 2009.

[13] E. Lavretsky and K. A. Wise, Robust and Adaptive Control With Aerospace Applications. Springer-Verlag, London, ISBN: 978-14471-4395-6 (Print) 978-1-4471-4396-3 (Online), 2013.

[14] R. Marino and P. Tomei, "Adaptive Control of Linear Time-Varying Systems," Automatica, vol. 39, pp. 651 - 659, 2003.

[15] R. H. Middleton, G. C. Goodwin, D. J. Hill, and D. Q. Mayne, "Design Issues in Adaptive Control," IEEE Trans. Autom. Contr., vol. 33, pp. $50-58,1988$.

[16] D. E. Miller and N. Mansouri, "Model Reference Adaptive Control Using Simultaneous Probing, Estimation, and Control," IEEE Trans. Autom. Contr., vol. 55, no. 9, pp. 2014 - 2029, September 2010.

[17] S. M. Naik, P. R. Kumar, and B. E. Ydstie, "Robust ContinuousTime Adaptive Control by Parameter Projection," IEEE Trans. Autom. Contr., vol. 37, no. 2, pp. 182 - 197, 1992.

[18] K. Narendra and A. Annaswamy, Stable Adaptive Control. Prentice Hall, 1989.

[19] N. Nguyen, K. Krishnakumar, J. Kaneshige, and P. Nespeca, "Flight Dynamics and Hybrid Adaptive Control of Damaged Aircraft," AIAA Journal of Guidance, Control, and Dynamics, vol. 31, no. 3, pp. 751764, 2008.

[20] J. B. Pomet and L. Praly, " Adaptive Nonlinear Regulation: Estimation from the Lyapunov Equation," IEEE Trans. Autom. Contr., vol. 37, no. 6, pp. 729-740, 1992.

[21] L. Praly, "Robust Model Reference Adaptive Controllers: Part I: Stability Analysis," In Proc. of the IEEE Conference on Decision and Control, 1984.

[22] S. S. Sastry and M. Bodson, Adaptive Control: Stability, Convergence and Robustness. Prentice Hall, 1989.

[23] V. Stepanyan and K. Krishnakumar, "MRAC Revisited: Guaranteed Performance with Reference Model Modification," In Proc. of the American Control Conference, Baltimore, NJ, June 2010.

[24] — "Indirect M-MRAC for Systems with Time Varying Parameters and Bounded Disturbances," In Proc. of the IEEE Multi-Conference on Systems and Control, Dubrovnik, Croatia, 2012.

[25] L. Vu and D. Liberzon, "Supervizory Control of Uncertain Linear Time-Varying Systems," IEEE Trans. Autom. Contr., vol. 55, no. 1, pp. 27 - 42, January 2011.

[26] K. A. Wise, E. Lavretsky, and N. Hovakimyan, "Adaptive Control of Flight: Theory, Applications, and Open Problems," In Proceedings of the American Control Conference, Minneapolis, MN, USA, pp. 59675971, June 14-16, 2006. 